\title{
Different culture media affect growth characteristics, surface marker distribution and chondrogenic differentiation of human bone marrow-derived mesenchymal stromal cells
}

\author{
Sebastien Hagmann ${ }^{1}$, Babak Moradi ${ }^{1}$, Sebastian Frank${ }^{1}$, Thomas Dreher ${ }^{1}$, Peer Wolfgang Kämmerer ${ }^{2}$, \\ Wiltrud Richter ${ }^{3}$ and Tobias Gotterbarm ${ }^{1 *}$
}

\begin{abstract}
Background: Bone marrow-derived mesenchymal stromal cells (BM-MSCs) play an important role in modern tissue engineering, while distinct variations of culture media compositions and supplements have been reported. Because MSCs are heterogeneous regarding their regenerative potential and their surface markers, these parameters were compared in four widely used culture media compositions.

Methods: MSCs were isolated from bone marrow and expanded in four established cell culture media. MSC yield/1000 MNCs, passage time and growth index were observed. In P4, typical MSC surface markers were analysed by fluorescence cytometry. Additionally, chondrogenic, adipogenic and osteogenic differentiation potential were evaluated.

Results: Growth index and PO cell yield varied importantly between the media. The different expansion media had a significant influence on the expression of CD10, CD90, CD105, CD140b CD146 and STRO-1. While no significant differences were observed regarding osteogenic and adipogenic differentiation, chondrogenic differentiation was superior in medium A as reflected by GAG/DNA content.
\end{abstract}

Conclusions: The choice of expansion medium can have a significant influence on growth, differentiation potential and surface marker expression of mesenchymal stromal cells, which is of fundamental importance for tissue engineering procedures.

Keywords: Mesenchymal stromal cells, Expansion media, Surface markers, Osteogenic differentiation, Chondrogenic differentiation, Adipogenic differentiation

\section{Background}

Since their recognition in the 1960s and 70s [1,2], mesenchymal stem or stromal cells (MSC) are considered one of the most promising targets for regenerative medicine. MSC were first isolated from animal bone marrow (BM) [3], but a variety of tissues in humans, such as adipose tissue [4], cord blood [5], peripheral blood [6] and connective tissues [7] have also proven to be a yielding source for these cells.

\footnotetext{
* Correspondence: tobias.gotterbarm@med.uni-heidelberg.de 'Department of Orthopedics, Trauma Surgery and Spinal Cord Injury, University Hospital Heidelberg, Germany Schlierbacher Landstrasse 200a, 69118 Heidelberg, Germany

Full list of author information is available at the end of the article
}

The potency of human MSC (hMSC) to differentiate into various cell lines, such as fibroblasts, myofibroblasts, osteoblasts, chondroblasts and adipocytes [8,9] put these cells into the current focus of tissue engineering, particularly in the fields of bone $[10,11]$ and cartilage $[12,13]$ regeneration, but also in myocardial infarction [14,15].

Minimal criteria for MSC have been postulated by the International Society for Cellular Therapy [16]: they must express CD105, CD73 and CD90 and lack expression of hematopoietic markers such as CD45, CD34, CD14 and CD11b. In addition, MSC must be capable of differentiating into fibroblasts, osteoblasts, adipocytes and chondroblasts under specific in vitro conditions. 
However, there is an enormous variance in cell isolation techniques and expansion conditions for MSCs [12,17-19]. Basal culture media and supplements have become a considerable market with the growth of mesenchymal stromal cell research. Up to this day, only little attention was paid to the distinct variations of culture media, growth factors and other supplements used in this field of research.

Yet there is increasing evidence that the choice of culture media has an important influence on the biological properties of MSCs [9,19-21]. Besides, recent studies report that bone marrow derived MSC may consist of several distinct subpopulations with diverse regenerative potential $[22,23]$. However, the properties of these subpopulations and their usability in tissue engineering are only marginally explored.

The aim of this study therefore was to analyse the influence of four different widely used culture media on growth parameters and surface marker distribution of human BMMSCs. Rather than determining the influence of single culture additives, actual media compositions that were in use in our laboratory were compared to each other with the main focus on the impact on surface marker distribution.

\section{Methods}

\section{Bone marrow donors}

Human MSCs were isolated from bone marrow of 12 donors (mean age $33 \pm 21.6$ years, 6 female, 6 male). Bone marrow aspiration was performed in the tibia $(n=2)$, femur $(n=2)$ or the iliac crest $(n=8)$ within the framework of total joint arthroplasty or osteotomy. The study protocol had been approved by the ethics committee of the University of Heidelberg, Germany. All patients provided informed consent according to the latest version of the Helsinki Declaration.

\section{Mesenchymal stromal cells (MSCs)}

Bone marrow was intraoperatively diluted to $1: 1$ isotonic saline solution (B. Braun Melsungen, Germany) with 5000 I.E. of heparine (ratiopharm, Ulm, Germany). Mononuclear cells (MNCs) were isolated from these bone marrow samples by Ficoll Paque plus (GE Healthcare, Uppsala, Sweden) gradient centrifugation. After Ficoll separation, the cells of all donors were distributed into four equal samples and resuspended in different cell culture media as described below at a density of $5 \times 10^{5}$ cells $/ \mathrm{cm}^{2}$ (equals $2.5 \times 10^{6}$ cells $/ \mathrm{ml}$ ) in $\mathrm{T} 75 \mathrm{~cm}^{2}$ cell culture flasks (Nunc, Roskilde, Denmark). MSCs were then cultured under the exact same conditions, at $37^{\circ} \mathrm{C}$ with $6 \% \mathrm{CO} 2$ in a humidified thermostat, except for the use of four different culture media:

1. Dulbecco's modified Eagle's medium low glucose (DMEM-LG, Invitrogen, Karlsruhe, Germany) with 10\% fetal calf serum (FCS, Biochrom, Berlin, Germany) and 1\% penicilline/streptomycine (Invitrogen, Karlsruhe, Germany) (referred to as medium A).

2. Alpha minimum essential medium ( $\alpha$ MEM) with L-glutamine with $10 \%$ fetal calf serum (FCS, Biochrom, Berlin, Germany) and $1 \%$ penicilline/streptomycine (Invitrogen, Karlsruhe, Germany) (medium B).

3. A variation of "Verfaillie" medium (medium C) [18], consisting of $547.5 \mathrm{ml}$ Dulbecco's modified Eagle's medium high glucose (DMEM-HG, Invitrogen, Karlsruhe, Germany) and L-glutamine (Invitrogen, Karlsruhe, Germany), $40 \mathrm{ml}$ MCDB (Sigma-Aldrich, Steinheim, Germany), $20 \mathrm{ml}$ FCS (Biochrom, Berlin, Germany), $10 \mathrm{ml}$ penicilline/ streptomycine (Invitrogen, Karlsruhe, Germany), $20 \mathrm{ml}$ IST (Sigma-Aldrich, Steinheim, Germany), 2 ml dexamethasone (Sigma-Aldrich, Steinheim, Germany), $500 \mu \mathrm{l}$ ascorbic acid (Sigma-Aldrich, Steinheim, Germany), 10 ng/ml PDGF-BB (Miltenyi Biotec, Bergisch Gladbach, Germany), $10 \mathrm{ng} / \mathrm{ml}$ EGF (Miltenyi Biotec, Bergisch Gladbach, Germany).

4. A variation of "Bernese chondrocyte medium" (medium D) [24,25], consisting of Dulbecco's modified Eagle's medium F12 + L-glutamine (Invitrogen, Karlsruhe, Germany), 1\% penicilline/streptomycine (Invitrogen, Karlsruhe, Germany), 10\% FCS (Biochrom, Berlin, Germany), $10 \mu \mathrm{l} / 50 \mathrm{ml}$ TGF-ß1 (Acris, Herford, Germany), $2.5 \mu \mathrm{l} / 50 \mathrm{ml}$ FGF-2 (Acris, Herford, Germany).

After 24 hours, non-adherent cells were discarded; afterwards, medium replacement was carried out every 72 hours.

Cells were inspected daily for confluence by polarisation microscopy. When reaching $80 \%$ confluence, cells were detached by incubation with trypsine (Biochrom, Berlin, Germany), harvested and washed with the corresponding complete medium. Cells were counted in triplicates after staining with trypan blue $0.4 \%$ (Sigma-Aldrich, Steinheim, Germany). MSCs were then resuspended and further cultured according to the protocol detailed above. The end of this first passage was defined as P1. The procedure was repeated until the end of the fourth passage (P4).

\section{Growth parameters}

Cells were counted before and after Ficoll separation, and after each passage in triplicates as detailed above, and passage time was analyzed. The following parameters were then calculated: MSC yield in P0, P1, P2, P3 and P4 per 1000 BM-MNCs and MSC growth index (MSCs before passage $\mathrm{x} / \mathrm{MSCs}$ after passage $\mathrm{x}$ ) and compared between the media.

\section{Chondrogenic differentiation}

After P4, MSCs were harvested with trypsine/EDTA as described above and centrifuged into pellets containing $5 \times 10^{5}$ cells $(n=6$ donors per group, $n=3-5$ pellets per 
donor). Chondrogenic differentiation was induced with a chondrogenic medium consisting of $286 \mathrm{ml}$ DMEM HG (Invitrogen, Karlsruhe, Germany), $150 \mu \mathrm{l}$ transferrin $10 \mathrm{mg} / \mathrm{ml}$ (Sigma-Aldrich, Steinheim, Germany), $1 \mu \mathrm{l}$ sodium selenite $100 \mu \mathrm{g} / \mathrm{ml}$ (Sigma-Aldrich, Steinheim, Germany), $3 \mathrm{ml}$ Sodium pyruvate $350 \mathrm{mM}$ (SigmaAldrich, Steinheim, Germany), $5 \mathrm{ml} \mathrm{BSA} \mathrm{7.5 \%} \mathrm{(Invitrogen,}$ Karlsruhe, Germany), $3 \mathrm{ml}$ P/S (Invitrogen, Karlsruhe, Germany), supplemented with $50 \mu \mathrm{l}$ dexamethasone $/ 5 \mathrm{ml}$ (Sigma-Aldrich, Steinheim, Germany), $5 \mu \mathrm{l}$ ascorbic acid/5 ml (Sigma-Aldrich, Steinheim, Germany), $5 \mu \mathrm{l}$ TGF-ß1/5 ml (Acris, Herford, Germany), $6.9 \mu \mathrm{l}$ insuline glargin/5 ml (Sanofi Aventis, Frankfurt, Germany). Medium was changed three times a week. Chondrogenic differentiation potential was evaluated by a glycosaminoglycan (GAG) assay $(n=2$ per donor). The pellets were digested with pepsin solution overnight. 1,9-dimethyl-methylene blue (dye content 80\%, SigmaAldrich, Steinheim, Germany) was used for staining of glycosaminoglycans. Measurements of absorption were performed at $530 \mathrm{~nm}$ and compared to a chondroitin 4-sulfate standard (Sigma-Aldrich, Steinheim, Germany). DNA content was measured with Quant iT ds Pico Green DNA Assay Kit (Invitrogen, Karlsruhe, Germany) according to manufacturers' protocols.

In addition, the remaining pellets were fixed in $4 \%$ paraformaldehyde and cut into $5 \mu \mathrm{m}$ sections for histology and immunohistochemistry. The sections were either stained with $1 \%$ alcian blue (Chroma, Muenster, Germany) and counterstained with fast red (SigmaAldrich, Steinheim, Germany), SafraninO/Fast Green (Sigma-Aldrich, Steinheim, Germany) or used for immunohistochemistry. Collagen I and Collagen II staining was performed by pre-treating the sections with $2 \mathrm{mg} / \mathrm{ml}$ hyaluronidase (Merck, Darmstadt, Germany) and $1 \mathrm{mg} / \mathrm{ml}$ pronase (Roche Diagnostics, Penzberg, Germany). The sections were then subjected to PBS containing 5\% BSA in order to block unspecific background and incubated with a monoclonal mouse anti-human collagen type or II antibody (ICN Biomedicals, Aurora, OH) in PBS containing $1 \%$ BSA. Reactivity to the antibodies was detected by applying a biotinylated goat anti-mouse secondary antibody (Dianova, Hamburg, Germany), streptavidin-alkaline phosphatase (Dako, Hamburg, Germany), and fast red (SigmaAldrich, Steinheim, Germany).

\section{Osteogenic differentiation}

After passage 4, MSCs were harvested with trypsin/ EDTA as described above and 35,000 cells per well were seeded in 24 well plates (Nunc, Roskilde, Denmark) containing osteogenic induction medium which consisted of DMEM high glucose (Invitrogen, Karlsruhe, Germany), 10\% FCS (Biochrom, Berlin, Germany), $0.1 \mathrm{mM}$ dexamethasone (Sigma-Aldrich, Steinheim, Germany),
$0.17 \mathrm{mM}$ ascorbic acid 2-phosphate (Sigma-Aldrich, Steinheim, Germany), $10 \mathrm{mM} \beta$-glycerophosphate (SigmaAldrich, Steinheim, Germany) and $1 \%$ penicilline/ streptomycine (Biochrom, Berlin, Germany).

At day 21, osteogenesis was quantified by alkaline phosphatase assay according to standard protocols. Briefly, MSCs were lysed in $0.5 \mathrm{ml} \mathrm{1 \%}$ Triton X-100 (Sigma-Aldrich, Steinheim, Germany). $100 \mathrm{ml}$ lysate were incubated with $100 \mathrm{ml}$ of $1 \mathrm{mg} / \mathrm{ml} \mathrm{p}$-nitrophenylphosphate in ALP-buffer (0.1 M glycine, $1 \mathrm{mM} \mathrm{MgCl} 2,1 \mathrm{mM} \mathrm{ZnCl}$, $\mathrm{pH}$ 10.4). The substrate turnover was then measured at 405/490 nm using an MRX ELISA reader (Dynatech Laboratories, Stuttgart, Germany). Results were standardized to lysate protein content using a Micro BCA Protein Assay Kit (Pierce, Rockford, USA) according to manufacturers' instructions.

In addition, calcium deposition was quantified by 0.5\% Alizarin Red S staining (Chroma, Münster, Germany) according to standard protocols and quantified at $570 \mathrm{~nm}$. The results were standardized to whole protein content as described above.

\section{Adipogenic differentiation}

After passage 4, MSCs were harvested with trypsin/ EDTA as described above and 35,000 cells per well were seeded in 24 well plates (Nunc, Roskilde, Denmark) containing adipogenic induction medium, consisting of DMEM high glucose (Invitrogen, Karlsruhe, Germany), 10\% FCS (Biochrom, Berlin, Germany), $1 \mathrm{mM}$ dexamethasone (Sigma-Aldrich, Steinheim, Germany), $0.2 \mathrm{mM}$ indomethacine (Sigma-Aldrich, Steinheim, Germany), $0.5 \mathrm{mM}$ isobutyl methylxanthine (Sigma-Aldrich, Steinheim, Germany), $0.01 \mathrm{mg} / \mathrm{ml}$ insulin glargin (Sanofi-Aventis, Frankfurt, Germany) and $1 \%$ penicilline/streptomycine (Biochrom, Berlin, Germany).

At day 21 the cells were fixed with $4 \%$ paraformaldehyde and stained with $0.3 \%$ Oil Red O solution (Chroma, Münster, Germany) to quantify differentiation into adipocyte. Re-extraction of the dye was performed with $60 \%$ isopropanol and the optical density was measured at $490 \mathrm{~nm}$.

\section{Flow cytometry analysis}

MSCs were detached with trypsine as described above, washed in whole medium and resuspended in phosphate buffered saline with $0.5 \%$ FCS and 2 mM EDTA.

The following anti-human antibodies were used in the experiments: CD105 PE mouse IgG1, CD133 mouse IgG1 (Miltenyi Biotec, Bergisch Glattbach, Germany), CD10 FITC mouse IgG1, CD13 PE mouse IgG1, CD14 FITC mouse IgG1, CD34 PE mouse IgG1, CD44 FITC mouse IgG2b, CD45 FITC mouse IgG1, CD49a PE mouse IgG1, CD90 FITC mouse IgG1, CD140b PE mouse IgG2a CD146 PE mouse IgG1, CD166 PE mouse IgG1, CD271 mouse IgG1 (BD Biosciences, Heidelberg, Germany), 
CD340 mouse IgG1 (Genway, San Diego, USA), HLA-ABC PE (Dako, Glostrup, Denmark), for MSC analysis. STRO-1 mouse IgM ( $\mathrm{R}+\mathrm{D}$ Systems, Wiesbaden, Germany) was labeled with a secondary goat anti-mouse FITC antibody (Dako, Glostrup, Denmark). Isotype matched control antibodies (IgG1 FITC and PE, IgG2a FITC and PE, IG2b FITC, all Dako, Glostrup, Denmark) were used for assessment of background fluorescence.

One-color and two color cytometry was performed using a FACS Scan ${ }^{\circledR}$ analyser (BD Biosciences, San Jose, USA) and the Cellquest Pro ${ }^{\circledR}$ Software (BD Biosciences, San Jose, USA). Positive fluorescence was defined as any event above the background fluorescence, which was defined by a histogram cut-off where $99.5 \%$ of the events in isotype antibody labeled cells were considered negative. All results regarding flow cytometry are displayed as \% positive cells.

\section{Statistical analysis}

Statistical analysis was performed using the SPSS computer software (SPSS Inc. Released 2009. PASW Statistics for Windows, Version 18.0. Chicago: SPSS Inc.). A three steps testing of normal distribution was performed for each data set: 1. Graphic display (QQ-plot, histogram and box plot), 2. Ratio analysis, 3. Kolmorogov-Smirnov (with Lilliefors significance correction) and ShapiroWilks testing. Analyses of variance (ANOVA) followed by Bonferroni correction were performed to compare the results for parametric data (osteogenic differentiation results). For non-parametric data, Friedman tests were performed to compare the four groups, followed by Wilcoxon tests for the comparison of two groups for paired data (chondrogenic differentiation results). A Kruskal-Wallis test for the comparison of four groups, followed by Mann-Whitney-U-tests was performed for non-paired data (growth parameters, surface markers). Differences were considered statistically significant for p-values smaller 0.05 . Results are displayed as means \pm standard deviation.

\section{Results}

MSC growth characteristics are strongly influenced by the choice of expansion media

An important donor-dependency of MSC growth characteristics was observed. However, there was no apparent correlation between growth parameters and the age of the donor or the site of bone marrow harvest.

The amount of MNCs in the bone marrow aspirates ranged from $6.25 \times 10^{5} / \mathrm{ml}$ to $1.69 \times 10^{7} / \mathrm{ml}$ (mean $3.85 \pm$ $4.9 \times 10^{6}$ cells $\left./ \mathrm{ml}\right)$. Mean MSC yield per $10^{3} \mathrm{BM}-\mathrm{MNCs}$ at P0 was significantly higher in medium $\mathrm{C}$ when compared to medium A $(297.29 \pm 248.21$ in medium $C$ vs. $70.31 \pm$ 88.09 in medium A; $\mathrm{p}=0.018$ ) (Figure 1a). This was also reflected by a higher cell count and earlier confluency in light microscopy when cells cultured in medium $\mathrm{C}$ were compared to the other media at the same time points (Figure 2). Four MNC samples in medium A and D and one in medium $B$ and $C$ showed no growth at all.

Cell proliferation rate differed substantially between the media with significantly lower growth index values for medium A, B and D compared to medium C in P1 (Figure $1 \mathrm{~b}$, comparison of all groups $\mathrm{p}<0.001 ; 5.77 \pm$ 4.14 for medium $C$ vs. $2.16 \pm 1.22$ for medium $B, 2.2 \pm$ 1.02 for medium $\mathrm{A}, 3.9 \pm 1.27$ for medium $\mathrm{C} ; \mathrm{p}=0.0033$, $\mathrm{p}=0.0027$ and $\mathrm{p}=0.0087$, respectively). In $\mathrm{P} 2$, no significant differences between the media were observed regarding the growth index (comparison of all groups $\mathrm{p}=0.32$ ) due to the fact that proliferation in medium $\mathrm{C}$

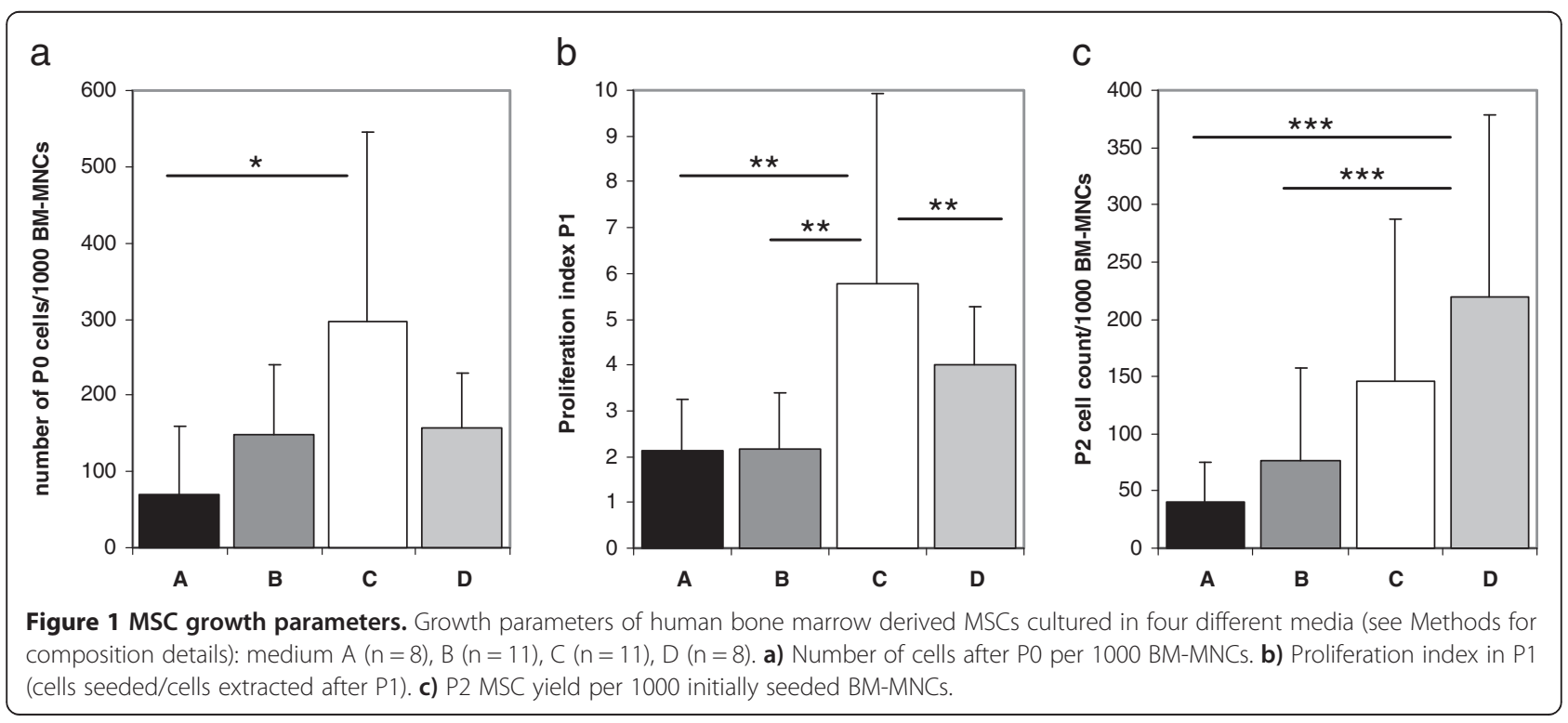



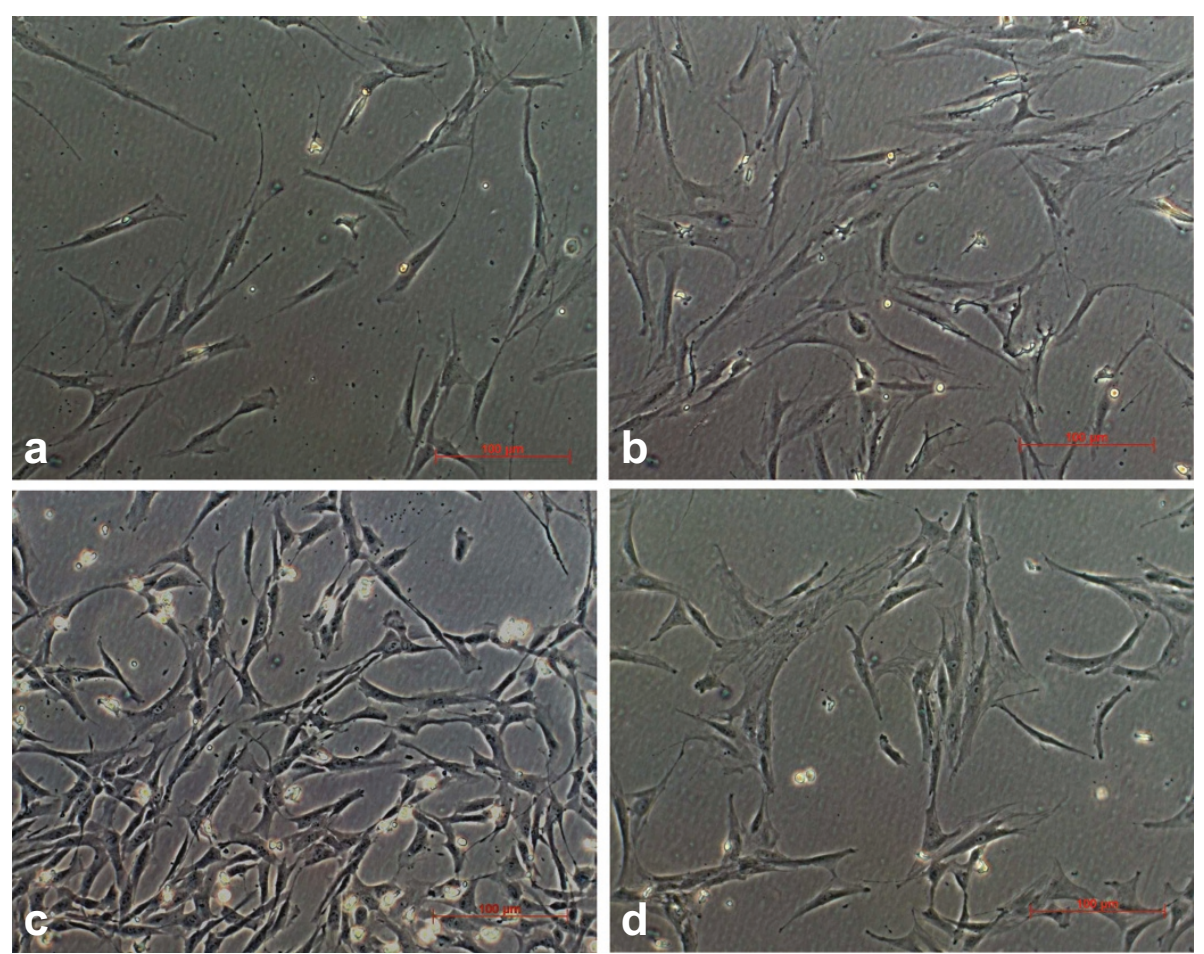

Figure 2 MSC morphology. Gross appearance of human BM-MSCs cultured in four different media after 8 days in P0, polarized light microscopy, index $100 \mu \mathrm{m}$. a) medium A b) medium B c) medium C d) medium D (see Methods for media composition). While all media display a similar cell phenotype typical for plastic adherent MSCs, MSCs cultured in medium C grew faster with earlier confluence.

was significantly reduced in P2 when compared to P1 $(2.35 \pm 1.57$ in $\mathrm{P} 2$ vs. $5.77 \pm 4.14$ in $\mathrm{P} 1, \mathrm{p}=0.014)$. Interestingly, this resulted in medium $\mathrm{D}$ showing the highest cell yield per $1000 \mathrm{BM}-\mathrm{MNCs}$ after P2 (Figure 1c, $219.37 \pm$ 158.32 for medium $\mathrm{D}$ vs. $41.14 \pm 33.9$ for medium $\mathrm{A}$, $\mathrm{p}<0.001$; vs. $76.54 \pm 81.52$ for medium $\mathrm{B}, \mathrm{p}=0.005$; comparison of all groups $\mathrm{p}=0.01)$. No significant differences in proliferation were observed between any of the culture conditions tested after P2.

Passage time in P0 was significantly lower in medium C and medium $\mathrm{B}$ compared to medium A (comparison of all groups $\mathrm{p}=0.021 ; 12 \pm 2.13$ days in medium $\mathrm{C}$ and $12.75 \pm$ 2.34 days in medium $\mathrm{B}$ vs. $17.58 \pm 5.98$ in medium $\mathrm{A}$, $\mathrm{p}=0.004$ and $\mathrm{p}=0.012$, respectively). Although medium $\mathrm{C}$ showed the lowest mean passage time in $\mathrm{P} 1$, this trend did not reach significance (data not shown, $\mathrm{p}=0.096$ ). In $\mathrm{P} 2$, medium $\mathrm{C}$ showed the fastest passage time with a significant difference to medium B (3.91 \pm 1.3 days vs. $7.25 \pm$ 2.63 days, $\mathrm{p}<0.001$; comparison of all groups $\mathrm{p}=0.003$ ). Regarding passage time, no significant differences were observed after P2.

\section{Distribution of several BM-MSCs surface markers is dependent on expansion media}

Fluorescence cytometry of MSC surface markers allowed distinction into clearly "positive", clearly "negative" and intermediate MSC markers (Table 1, Figure 3). In all media, there was a strong fluorescence signal ("positive" markers) for CD13, CD44, CD73, CD90, CD105 and CD166 (Table 1, Figure 3). There was an important variation of CD105 fluorescence in medium $C$, resulting in a significantly lower mean fluorescence compared to the other media (comparison of all groups $\mathrm{p}=0.0011$; $72.69 \pm 20.72 \%$ in medium C vs. $98.29 \pm 2.04 \%$ in medium $\mathrm{B}, \mathrm{p}=0.012$ and $98.79 \pm 1.22 \%$ in medium $\mathrm{D}$, $\mathrm{p}=0.015)$. A significantly reduced fluorescence for CD90 was also found in medium $\mathrm{C}$ when compared to medium $\mathrm{B}$ and medium $\mathrm{D}$ (comparison of all groups $\mathrm{p}=0.001,91.89 \pm 3.65 \%$ for medium $\mathrm{C}$ vs. $99.12 \pm 2.24 \%$ for medium $\mathrm{B}, \mathrm{p}=0.003$ and $98.77 \pm 1.39 \%$ for medium $\mathrm{D}$; $\mathrm{p}=0.002$, respectively). CD166 fluorescence was reduced in medium $C$ when compared to medium $D(p=0.026)$. There was no difference between the media regarding CD13, CD44 and CD73 fluorescence.

Low fluorescence ("negative" markers) was observed for CD10, CD14, CD34, CD45, CD133 and CD271 (Table 1). No significant differences between the media were observed except for CD10 fluorescence in medium C compared to medium B (Figure 3, $26 \pm 21.84 \%$ vs. $1.02 \pm$ $1.35 \%, \mathrm{p}=0.01$ ).

Numerous surface markers were found to be highly donor dependent (CD49, CD140b, CD146, CD340 and 
Table 1 Surface marker distribution on human bone marrow derived mesenchymal stromal cells in four different culture conditions

\begin{tabular}{ccccc}
\hline \multicolumn{1}{c}{ Medium A } & Medium B & Medium C & Medium D \\
\hline CD10 & $12.954 \pm 18.76$ & $1.02 \pm 1.35$ & $26 \pm 21.84$ & $3.15 \pm 3.44$ \\
CD13 & $95.37 \pm 8.08$ & $98.97 \pm 2.03$ & $99.83 \pm 0.11$ & $99.79 \pm 0.27$ \\
CD14 & $0.36 \pm 0.19$ & $1.21 \pm 1.28$ & $0.47 \pm 0.22$ & $0.63 \pm 0.2$ \\
CD34 & $0.40 \pm 0.42$ & $0.31 \pm 0.25$ & $0.32 \pm 0.13$ & $0.34 \pm 0.15$ \\
CD44 & $94.04 \pm 8$ & $98.27 \pm 1.99$ & $96.13 \pm 4.47$ & $99.22 \pm 0.6$ \\
CD45 & $0.342 \pm 0.22$ & $0.33 \pm 0.18$ & $0.36 \pm 0.17$ & $0.2 \pm 0.16$ \\
CD49 & $52.03 \pm 22.36$ & $61.37 \pm 18.11$ & $45.15 \pm 31.27$ & $48.23 \pm 23.98$ \\
CD73 & $95.572 \pm 4.19$ & $98.1 \pm 1.95$ & $97.57 \pm 2.71$ & $99.58 \pm 0.26$ \\
CD90 & $95.25 \pm 5.68$ & $99.12 \pm 2.24$ & $91.89 \pm 3.65$ & $98.77 \pm 1.39$ \\
CD105 & $92.32 \pm 6.98$ & $98.29 \pm 2.04$ & $72.69 \pm 20.72$ & $98.79 \pm 1.22$ \\
CD133 & $0.57 \pm 0.32$ & $0.71 \pm 1.1$ & $0.74 \pm 0.15$ & $0.68 \pm 0.61$ \\
CD140b & $70.42 \pm 34.91$ & $90.51 \pm 11.44$ & $10.87 \pm 15$ & $78.12 \pm 25$ \\
CD146 & $47.64 \pm 29.68$ & $47.64 \pm 28.96$ & $19.27 \pm 28.6$ & $84.92 \pm 9.51$ \\
CD166 & $90.49 \pm 12.19$ & $93.21 \pm 9.34$ & $90.16 \pm 8.11$ & $99.25 \pm 0.81$ \\
CD271 & $1.08 \pm 1.03$ & $1.8 \pm 2.71$ & $2.68 \pm 2.34$ & $2.69 \pm 2.6$ \\
CD340 & $44.4 \pm 36.34$ & $69.96 \pm 32.97$ & $39.31 \pm 24.86$ & $68.61 \pm 35.31$ \\
STRO-1 & $48.26 \pm 32.42$ & $78.11 \pm 27.47$ & $42.07 \pm 17.09$ & $67.07 \pm 36.24$ \\
HLA-ABC & $92.02 \pm 17.18$ & $97.13 \pm 5.38$ & $90.62 \pm 10.83$ & $99.76 \pm 0.17$ \\
\hline Resut a & & &
\end{tabular}

Results are displayed as means of \% positive cells \pm standard deviation.

STRO-1), however, despite this fact, significant differences between the media were observed.

CD146 showed intermediate fluorescence in both medium A and medium B with about $50 \%$ of the cells being positive for this marker. Of all media, medium $\mathrm{C}$ showed the lowest expression of CD146 while expression was significantly higher in medium D (comparison of all groups $\mathrm{p}=0.005 ; 84.92 \pm 9.51 \%$ in medium $\mathrm{D}$ vs. $19.27 \pm$ $28.6 \%$ in medium $\mathrm{C}, \mathrm{p}=0.001 ; 47.64 \pm 29.68$ in medium $\mathrm{A}$, $\mathrm{p}=0.003 ; 47.64 \pm 29.96$ in medium $\mathrm{B}, \mathrm{p}=0.016$; Figure 3 ). CD140b expression was also found to be significantly lower in medium $\mathrm{C}$ when compared to the other media (comparison of all groups $\mathrm{p}=0.003 ; 10.87 \pm 15 \%$ vs. $70.42 \pm 35 \%$ in medium $\mathrm{A}, \mathrm{p}=0.007 ; 90.51 \pm 11.44 \%$ in medium $\mathrm{B}, \mathrm{p}<0.001 ; 78.12 \pm 25 \%$ in medium $\mathrm{D}, \mathrm{p}=0.004$; Figure 3). No significant differences were observed for CD49 and CD340 (Table 1). There was also a lower STRO-1 expression in medium $C$ when compared to medium B (comparison of all groups $\mathrm{p}=0.047 ; 78.11 \pm$ $27.47 \%$ in medium B vs. $42.07 \pm 17.09 \%$ in medium C, $\mathrm{p}=0.022$; Figure 3 ).

The choice of expansion media affects MSC chondrogenic differentiation potential

In all media, MSCs were able to differentiate into chondrogenic, osteogenic and adipogenic lineages (Figures 4 and 5). No significant differences between the media were observed regarding osteogenic differentiation (Table 2; Alizarin per well $\mathrm{p}=0.455$, ALP per protein $\mathrm{p}=0.48$ ) Pellet size was significantly lower in medium $C(0.14 \pm$ $0.017 \times 10^{6}$ pixel) when compared to medium $B$ (Figure $5 \mathrm{a}$, $0.2 \pm 0.033 \times 10^{6}$ pixel, $\left.\mathrm{p}=0.028\right)$ and medium $\mathrm{D}(0.27 \pm$ $0.086 \times 10^{6}$ pixel, $\mathrm{p}=0.046$ ) and highest in medium $\mathrm{A}$, yet there was no significant difference between this medium and the other media. Chondrogenic differentiation as reflected by GAG/DNA content in the pellets was highest in medium A compared to the other media and lowest in medium $\mathrm{C}$, the differences however were only significant between medium $\mathrm{A}$ and $\mathrm{B}$ $(4786.11 \pm 4523.34$ in medium A vs. $1424.87 \pm 975.83$ in medium $B, p=0.046$; Figure 5 ). This difference in chondrogenic differentiation between the media was also reflected by histology (Figure 4c), although Safranin $\mathrm{O} /$ Fast Green staining revealed donor-dependent variations in all groups.

\section{Discussion}

After less than two decades of basic research on their proliferation and differentiation potential as well as their immunosuppressive properties, MSCs have entered clinical applications in regenerative medicine, with numerous clinical trials in the fields of orthopaedics, hematology, neurology, cardiology, dentistry and in the treatment of genetical disorders [26-31]. However, there are important variations of isolation and expansion techniques throughout each laboratory, and reports of MSC subtypes with distinct properties are increasing [22,23]. In this context, our study investigated the effect of different currently used expansion media on proliferation, differentiation, and most importantly on surface marker distribution of human bone marrow derived MSCs.

Our data clearly demonstrate that the choice of expansion media can significantly alter not only BM-MSC growth characteristics, but also their differentiation potential such as the ability to form cartilaginous tissue in vitro. Our data are in line with findings of previous studies that have demonstrated inferior chondrogenic differentiation results of MSCs expanded in a control medium ("Verfaillie", which corresponds to medium C in our study) when compared to ES medium [21]. While ALP per protein was lower in medium $C$ than the other media, no significant differences could be observed between the media, which on the one hand can be explained by the high variation among the donors, and on the other hand by a generally poor osteogenic differentiation in our population. The same could be observed for adipogenic differentiation, which was generally poor, while variations among the donors could be observed. The reasons for these poor differentiation results may lie within the donors or the media preparations. However, all cell preparations were treated in the exact same way, 


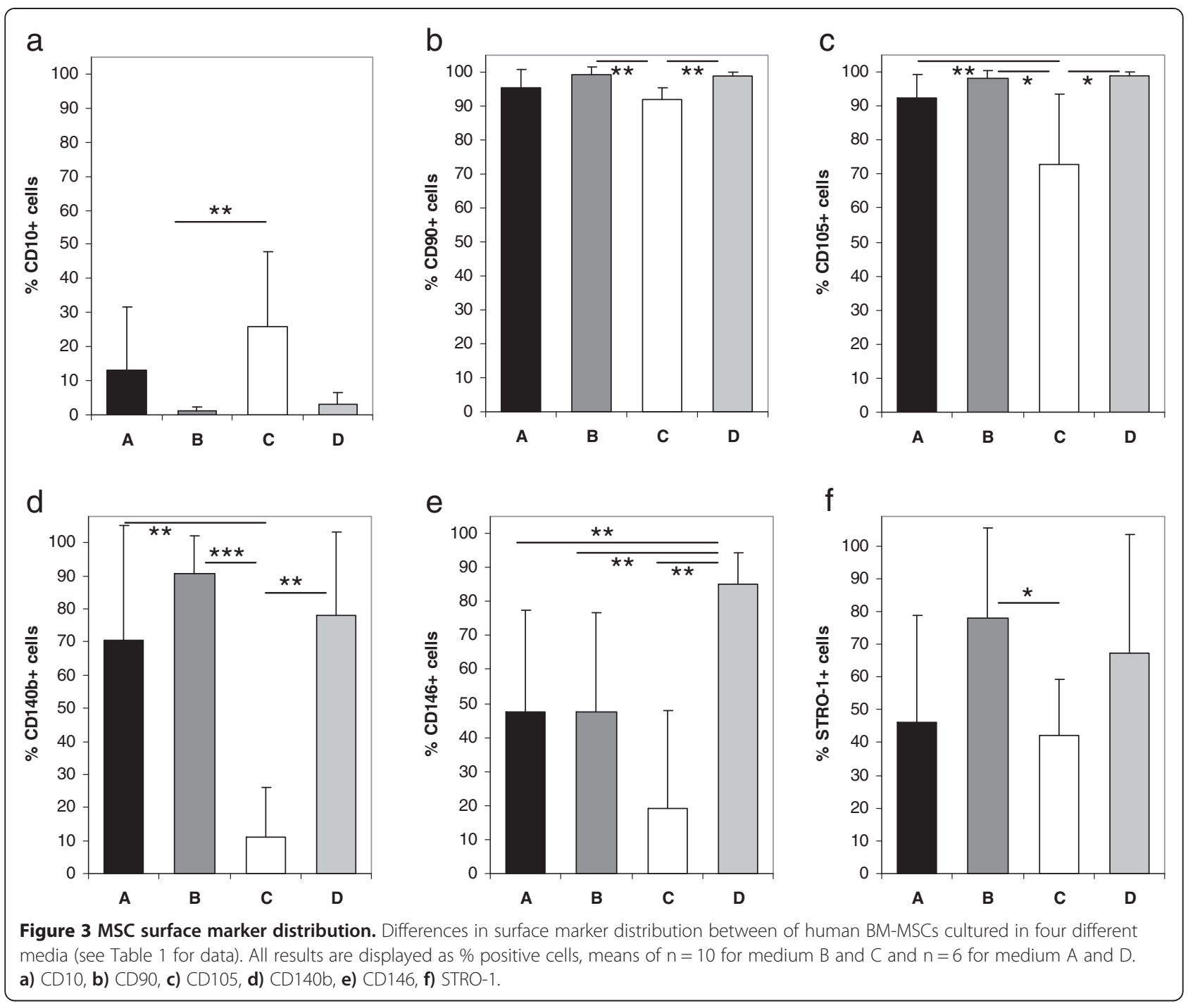

thus indicating that in this context, chondrogenic differentiation potential is more affected by the choice of expansion media than adipogenic and osteogenic differentiation.

Our study adds to findings that quantify differences in surface marker expression depending on the expansion media applied [32]. An important study in this field was published in 2006, revealing the impact not only of the choice of expansion media and growth factors, but also of basic factors such as plating density and flask manufacturer on MSC characteristics [19]. The experiments conducted in this study also revealed that certain MSC surface markers (CD44, MAB1470, STRO-1 and HLA-DR) showed intermediate fluorescence when MSCs were expanded in DMEM-HG, however without quantifying these results. Our study reveals characteristic changes in the distribution of CD10, CD140b and CD146 that to our best knowledge have not been published yet, with consistent results of some more stable markers such as CD14, CD34 and CD45 as "negative markers" and CD13, CD44, CD73 and CD166 as "positive" markers [33].

Another recent study has compared four different expansion media (among these DMEM and Alpha-MEM) and analysed adipose-tissue derived MSCs regarding their surface markers, concluding that the expression of CD49d, CD54 (being lowest in DMEM-KO) and CD117 was inconsistent at all passages and in all four media while the other surface markers did not significantly alter between the media [34]. This is not in accordance with other studies [32] and our own findings that clearly indicate significant differences in the expression of CD10 (not tested in the above study), CD90, CD105, CD140b (not tested in the above study) and CD146 (not tested in the above study). This discrepancy could be either due to differences in the study population or by assuming different characteristics of adipose-tissue derived 

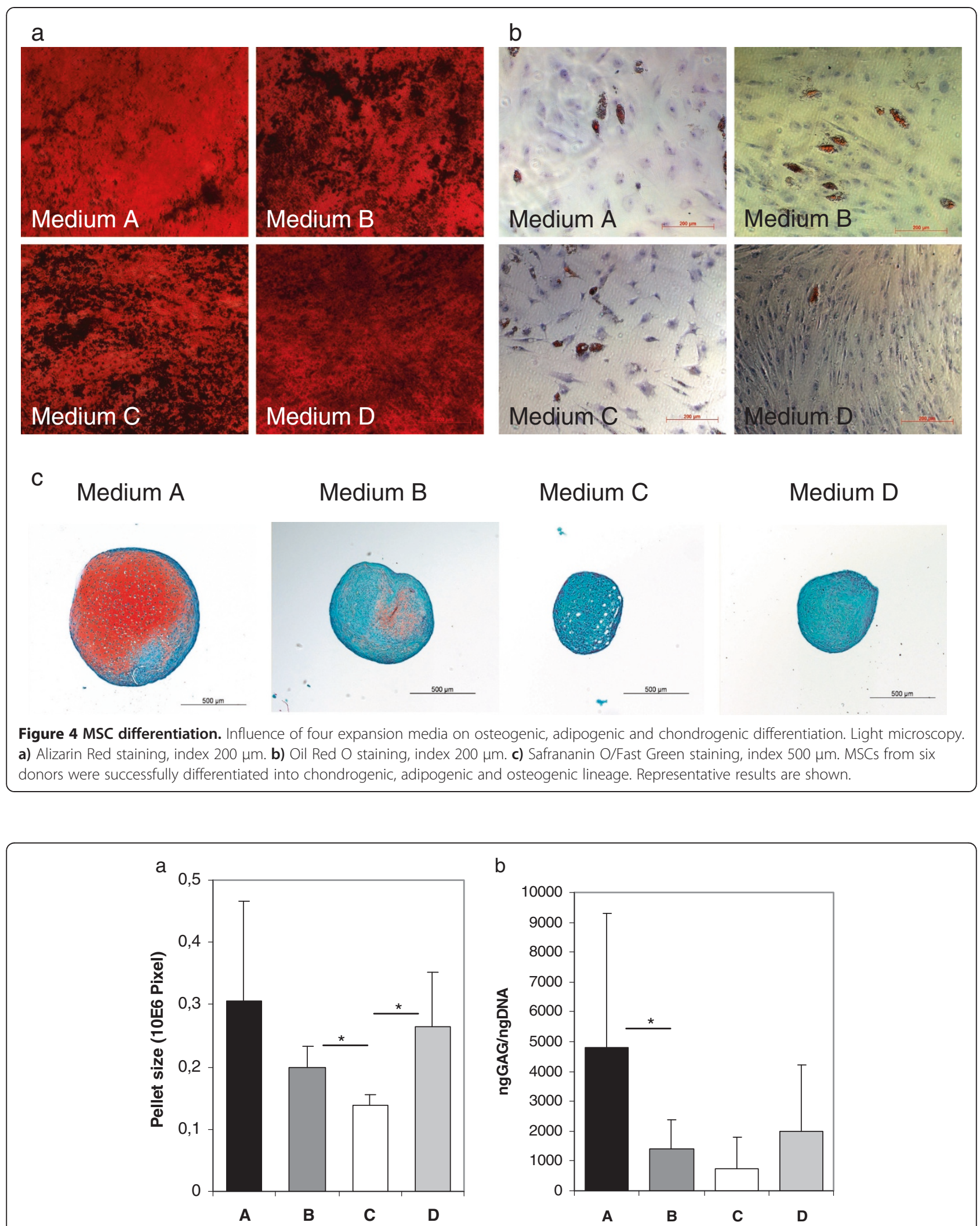

Figure 5 MSC chondrogenic differentiation. Chondrogenic differentiation results of human BM-MSCs cultured in different media. a) Pellet size after expansion in four different media, $n=6$ donors per group, experiments conducted in duplicates. $\mathbf{b}$ ) GAG/DNA content in pellets after expansion in four different media, $\mathrm{n}=6$ donors per group, experiments conducted in duplicates. 
Table 2 Quantitative results of osteogenic differentiation of human bone marrow derived mesenchymal stromal cells expanded in four different culture conditions

\begin{tabular}{lcccc}
\hline & Medium A & Medium B & Medium C & Medium D \\
\hline $\boldsymbol{\mu g}$ Alizarin/well & $174.96 \pm 67.47$ & $235.6 \pm 98.79$ & $242.48 \pm 145.24$ & $285.19 \pm 147.29$ \\
ALP per protein & $1.29 \pm 1.38$ & $1.42 \pm 0.95$ & $0.26 \pm 0.42$ & $0.76 \pm 0.48$ \\
\hline
\end{tabular}

Results are displayed as means \pm standard deviation.

stem cells [35-37], as well as by the lower number of donors $(n=3)$ used in the above study. Differences may also be due to the fact that dot charts were employed to analyse fluorescence without indication of a quantitative threshold. In our study, histograms and a threshold of 99.5\% were employed to determine positive fluorescence, thus allowing to unmask subtle differences in fluorescence between the media. Another reason for the discrepancy between both studies may be the use of growth factors in two of our media, revealing an important notion of how expansion media may pre-determine the selection of distinct subtypes of MSC through the use of growth factors.

Growth factors such as FGF-2 (or bFGF) and TGF- $ß$ have a long tradition in tissue engineering for modifying the differentiation potential of MSCs. For instance, expansion in the presence of FGF-2 is known to increase proliferation and chondrogenic potential of MSCs [38-40], but to inhibit chondrogenesis when applied during chondrogenic differentiation [41]. Application of FGF-2 to human BM-MSCs during expansion was also found to enhance osteogenesis and adipogenesis while suppressing neuronal differentiation [19]. TGF- $\beta$ isoforms have been extensively investigated for their potential to enhance chondrogenesis and osteogenesis [42-44].

However, in our experiments, the highest chondrogenic potential was obtained in a non-growth factor enhanced medium (medium A). This controversy may be due to the fact that differentiation was induced after P4. It is known that the influence of growth factors diminishes with higher passages [38]. Also, the effect cannot be extrapolated on presence or absence of growth factors, as all tested media contained fetal calf serum, which shows such a high variability that most groups select specifically pre-screened lots. In our experiments, the same FCS lot was used for expansion in all four culture conditions. Three of the four media contained 10\% FCS; however, growth indices and P0 cell yield/1000 BM-MNCs were highest in the variation of medium $\mathrm{C}$ containing $2 \%$ FCS, which may be due to its growth factor content. Although the growth factor content may also vary with different FCS lots, in this particular setting we thus directly could demonstrate that application of growth factors does not guarantee control of differentiation.

The use of these easy-to-handle bovine components also remains controversial due to their potential safety risks. Although several reports have propagated easy and efficient use of serum-free or xeno-free MSC expansion media $[17,45,46]$, none of these media have made it to a wide distribution, somewhat reflecting our own experience that the use of these media is still not as practicable as advertised.

The importance of our results lies within the notion that the quality of MSC application may be deeply altered by factors such as the choice of expansion media and the application of growth factors. Moreover, despite ongoing attempts of characterising MSCs and their subtypes, these cells still represent some sort of "black box" hiding properties that are not yet fully understood. It would seem that this knowledge should urge scientist to produce more reproducible results. However, apart from a few studies, no general attempts have been made to standardize MSC isolation and expansion procedures, although it is increasingly understood that MSC may exert different characteristics depending on which laboratory they are cultured in [47]. The minimal MSC-defining criteria made by the International Society for Cellular Therapy [16] have served to narrow the term "mesenchymal stem cell", however, they lack clarification of a) what is considered presence or absence of typical surface markers and b) how differentiation results can be compared to each other in a quantitative way, considering that numerous reports have outlined that differentiation properties may vary with distinct subtypes of MSCs, expansion and differentiation protocols and different tissues MSCs are derived from.

\section{Limitations}

Our experiments were characterized by an important variation of most parameters dependent on the donor of MSCs. A limitation of this study may be that the age of the donors was widely scattered; however, the influence of age and comorbidities on MSC expansion and differentiation is most controversially discussed [48]. In a small series of patients, our own findings suggested that the choice of culture media was more important than age and comorbidity [49]. Recent reports with larger numbers of patients reveal that proliferation characteristics may decrease with age, but that their lineage choice remains unaltered [48]. Although age may have influenced our findings, by distributing MSCs of all patients and culturing them in all four media, we were 
able to detect differences that could be solely assigned to the choice of culture media. However, we were able to observe an important variation among the donors that could not be attributed to age. The heterogeneity of MSC preparations of different donors is a known problem [50] that may have had an important influence on growth parameters and differentiation, but surface markers as well.

It could be criticized that two media containing different growth factors were examined along with two media without growth factors and that three media contained $10 \%$ FCS while one contained 2\% FCS. However, in this study, our aim was to compare four media that are actually widely used in different laboratories rather than to determine the influence of each component. In our opinion, this approach creates a clearer image of how allegedly trivial decisions may have an influence on the outcome of an experiment. It is clear that in the future, experiments attributing the observed differences to single media components will have to be conducted.

\section{Conclusions}

This study adds to several reports that emphasize on how the choice of expansion media can influence not only growth characteristics, but also differentiation potential of MSCs. This study is in accordance with previous findings reporting differences in the expression of typical MSC markers depending on the media applied. We were able to detect additional MSC markers affected by the expansion conditions that to our best knowledge have not been reported yet. Our findings suggest that attempts to further investigate how different media components affect MSC characteristics are necessary. More importantly, our findings are in favour of pursuing standardized protocols in MSC isolation and expansion.

\section{Abbreviations}

Alpha-MEM: Alpha minimum essential medium; BM: Bone marrow; CD: Cluster of differentiation; DMEM-LG: Dulbecco's modified Eagle's medium low glucose; FCS: Fetal calf serum; FGF-2: Fibroblast growth factor 2; GAG: Glycosaminoglycans; hMSCs: Human mesenchymal stromal cells; MNCs: Mononuclear cells.

\section{Competing interests}

The authors declare that they have no competing interests.

\section{Authors' contributions}

$\mathrm{SH}, \mathrm{BM}, \mathrm{WR}$ and TG conceived of the study. SH drafted the manuscript. SH, TD, TG, BM and SF provided the bone marrow. SH and SF carried out the experiments. SF, WR, PWK and SH performed the statistical analysis. SH, WR, BM, TD, PWK and TG participated in study design and coordination and helped to draft the manuscript. All authors read and approved the final manuscript.

\section{Acknowledgements}

We would like to acknowledge Rene Wetzel, Patrick Göthlich, Marc Hoffmann and Elena Tripel for their support. The study was carried out with funding by the state of Baden-Württemberg, Germany. None of the authors received external funding in connection with the study presented in this publication.

\section{Author details}

'Department of Orthopedics, Trauma Surgery and Spinal Cord Injury, University Hospital Heidelberg, Germany Schlierbacher Landstrasse 200a, 69118 Heidelberg, Germany. ${ }^{2}$ Maxillofacial and Plastic Surgery, University Medical Center, Mainz, Germany. ${ }^{3}$ Research Center for Experimental Orthopedics, University Hospital Heidelberg, Heidelberg, Germany.

Received: 22 April 2013 Accepted: 19 July 2013

Published: 30 July 2013

\section{References}

1. Becker AJ, Mc CE, Till JE: Cytological demonstration of the clonal nature of spleen colonies derived from transplanted mouse marrow cells. Nature 1963, 197:452-454.

2. Friedenstein AJ, Deriglasova UF, Kulagina NN, Panasuk AF, Rudakowa SF, Luria EA, Ruadkow IA: Precursors for fibroblasts in different populations of hematopoietic cells as detected by the in vitro colony assay method. Exp Hematol 1974, 2:83-92

3. Friedenstein AJ, Piatetzky S II, Petrakova KV: Osteogenesis in transplants of bone marrow cells. J Embryol Exp Morphol 1966, 16:381-390.

4. Zuk PA, Zhu M, Mizuno H, Huang J, Futrell JW, Katz AJ, Benhaim P, Lorenz HP, Hedrick MH: Multilineage cells from human adipose tissue: implications for cell-based therapies. Tissue Eng 2001, 7:211-228.

5. Bieback K, Kern S, Kluter H, Eichler H: Critical parameters for the isolation of mesenchymal stem cells from umbilical cord blood. Stem Cells 2004, 22:625-634.

6. Kuznetsov SA, Mankani MH, Gronthos S, Satomura K, Bianco P, Robey PG: Circulating skeletal stem cells. J Cell Biol 2001, 153:1133-1140.

7. da Silva ML, Chagastelles PC, Nardi NB: Mesenchymal stem cells reside in virtually all post-natal organs and tissues. J Cell Sci 2006, 119:2204-2213.

8. Jiang Y, Jahagirdar BN, Reinhardt RL, Schwartz RE, Keene CD, Ortiz-Gonzalez XR Reyes M, Lenvik T, Lund T, Blackstad M, et al: Pluripotency of mesenchymal stem cells derived from adult marrow. Nature 2002, 418:41-49.

9. Wagner W, Wein F, Seckinger A, Frankhauser M, Wirkner U, Krause U, Blake J, Schwager C, Eckstein V, Ansorge W, Ho AD: Comparative characteristics of mesenchymal stem cells from human bone marrow, adipose tissue, and umbilical cord blood. Exp Hematol 2005, 33:1402-1416.

10. Kallai I, van Lenthe GH, Ruffoni D, Zilberman Y, Muller R, Pelled G, Gazit D: Quantitative, structural, and image-based mechanical analysis of nonunion fracture repaired by genetically engineered mesenchymal stem cells. J Biomech 2010, 43:2315-2320.

11. Lyons FG, Al-Munajjed AA, Kieran SM, Toner ME, Murphy CM, Duffy GP O'Brien FJ: The healing of bony defects by cell-free collagen-based scaffolds compared to stem cell-seeded tissue engineered constructs. Biomaterials 2010, 31:9232-9243.

12. Goepfert C, Slobodianski A, Schilling AF, Adamietz P, Portner R: Cartilage engineering from mesenchymal stem cells. Adv Biochem Eng Biotechnol 2010, 123:163-200.

13. Guo J, Lin GS, Bao CY, Hu ZM, Hu MY: Anti-inflammation role for mesenchymal stem cells transplantation in myocardial infarction. Inflammation 2007, 30:97-104.

14. Hatzistergos KE, Quevedo H, Oskouei BN, Hu Q, Feigenbaum GS, Margitich IS, Mazhari R, Boyle AJ, Zambrano JP, Rodriguez JE, et al: Bone marrow mesenchymal stem cells stimulate cardiac stem cell proliferation and differentiation. Circ Res 2010, 107:913-922.

15. Mazhari R, Hare JM: Mechanisms of action of mesenchymal stem cells in cardiac repair: potential influences on the cardiac stem cell niche. Nat Clin Pract Cardiovasc Med 2007, 4(Suppl 1):S21-26.

16. Dominici M, Le Blanc K, Mueller I, Slaper-Cortenbach I, Marini F, Krause D, Deans R, Keating A, Prockop D, Horwitz E: Minimal criteria for defining multipotent mesenchymal stromal cells. The International Society for Cellular Therapy position statement. Cytotherapy 2006, 8:315-317.

17. Miwa H, Hashimoto Y, Tensho K, Wakitani S, Takagi M: Xeno-free proliferation of human bone marrow mesenchymal stem cells. Cytotechnology 2012, 64:301-308.

18. Reyes M, Lund T, Lenvik T, Aguiar D, Koodie L, Verfaillie CM: Purification and ex vivo expansion of postnatal human marrow mesodermal progenitor cells. Blood 2001, 98:2615-2625.

19. Sotiropoulou PA, Perez SA, Salagianni M, Baxevanis CN, Papamichail M: Characterization of the optimal culture conditions for clinical scale production of human mesenchymal stem cells. Stem Cells 2006, 24:462-471. 
20. Apel A, Groth A, Schlesinger S, Bruns H, Schemmer P, Buchler MW, Herr I: Suitability of human mesenchymal stem cells for gene therapy depends on the expansion medium. Exp Cell Res 2009, 315:498-507.

21. Dexheimer $V$, Frank S, Richter W: Proliferation as a requirement for in vitro chondrogenesis of human mesenchymal stem cells. Stem Cells Dev 2012, 21:2160-2169.

22. Buhring HJ, Battula VL, Treml S, Schewe B, Kanz L, Vogel W: Novel markers for the prospective isolation of human MSC. Ann N Y Acad Sci 2007, 1106:262-271

23. Buhring HJ, Treml S, Cerabona F, de Zwart P, Kanz L, Sobiesiak M: Phenotypic characterization of distinct human bone marrow-derived MSC subsets. Ann N Y Acad Sci 2009, 1176:124-134.

24. Barbero A, Grogan S, Schafer D, Heberer M, Mainil-Varlet P, Martin I: Age related changes in human articular chondrocyte yield, proliferation and post-expansion chondrogenic capacity. Osteoarthritis Cartilage 2004, 12:476-484

25. Grogan SP, Barbero A, Diaz-Romero J, Cleton-Jansen AM, Soeder S, Whiteside R, Hogendoorn PC, Farhadi J, Aigner T, Martin I, Mainil-Varlet P: Identification of markers to characterize and sort human articular chondrocytes with enhanced in vitro chondrogenic capacity. Arthritis Rheum 2007, 56:586-595.

26. Chen SL, Fang WW, Ye F, Liu YH, Qian J, Shan SJ, Zhang JJ, Chunhua RZ, Liao LM, Lin S, Sun JP: Effect on left ventricular function of intracoronary transplantation of autologous bone marrow mesenchymal stem cell in patients with acute myocardial infarction. Am J Cardiol 2004, 94:92-95.

27. ClinicalTrials.gov by the National Library of Medicine (NLM) at the National Institutes of Health (NIH). http:www.clinicaltrials.gov.

28. Horwitz EM, Gordon PL, Koo WK, Marx JC, Neel MD, McNall RY, Muul L, Hofmann T: Isolated allogeneic bone marrow-derived mesenchymal cells engraft and stimulate growth in children with osteogenesis imperfecta: Implications for cell therapy of bone. Proc Natl Acad Sci U S A 2002, 99:8932-8937.

29. Koc ON, Day J, Nieder M, Gerson SL, Lazarus HM, Krivit W: Allogeneic mesenchymal stem cell infusion for treatment of metachromatic leukodystrophy (MLD) and Hurler syndrome (MPS-IH). Bone Marrow Transplant 2002, 30:215-222.

30. Le Blanc K, Rasmusson I, Sundberg B, Gotherstrom C, Hassan M, Uzunel M, Ringden O: Treatment of severe acute graft-versus-host disease with third party haploidentical mesenchymal stem cells. Lancet 2004, 363:1439-1441.

31. Mazzini L, Fagioli F, Boccaletti R, Mareschi K, Oliveri G, Olivieri C, Pastore I, Marasso R, Madon E: Stem cell therapy in amyotrophic lateral sclerosis: a methodological approach in humans. Amyotroph Lateral Scler Other Motor Neuron Disord 2003, 4:158-161.

32. Haack-Sorensen M, Friis T, Bindslev L, Mortensen S, Johnsen HE, Kastrup J: Comparison of different culture conditions for human mesenchymal stromal cells for clinical stem cell therapy. Scand J Clin Lab Invest 2008, 68:192-203.

33. Fekete N, Gadelorge M, Furst D, Maurer C, Dausend J, Fleury-Cappellesso S, Mailander V, Lotfi R, Ignatius A, Sensebe L, et al: Platelet lysate from whole blood-derived pooled platelet concentrates and apheresis-derived platelet concentrates for the isolation and expansion of human bone marrow mesenchymal stromal cells: production process, content and identification of active components. Cytotherapy 2012, 14:540-554.

34. Dhanasekaran M, Indumathi S, Rashmi M, Rajkumar JS, Sudarsanam D: Unravelling the retention of proliferation and differentiation potency in extensive culture of human subcutaneous fat-derived mesenchymal stem cells in different media. Cell Prolif 2012, 45:516-526.

35. Strioga M, Viswanathan S, Darinskas A, Slaby O, Michalek J: Same or not the same? Comparison of adipose tissue-derived versus bone marrow-derived mesenchymal stem and stromal cells. Stem Cells Dev 2012, 21:2724-2752.

36. Vishnubalaji R, Al-Nbaheen M, Kadalmani B, Aldahmash A, Ramesh T: Comparative investigation of the differentiation capability of bonemarrow- and adipose-derived mesenchymal stem cells by qualitative and quantitative analysis. Cell Tissue Res 2012, 347:419-427.

37. Xie X, Wang Y, Zhao C, Guo S, Liu S, Jia W, Tuan RS, Zhang C: Comparative evaluation of MSCs from bone marrow and adipose tissue seeded in PRP-derived scaffold for cartilage regeneration. Biomaterials 2012, 33:7008-7018.

38. Cheng T, Yang C, Weber N, Kim HT, Kuo AC: Fibroblast growth factor 2 enhances the kinetics of mesenchymal stem cell chondrogenesis. Biochem Biophys Res Commun 2012, 426:544-550.
39. Stewart AA, Byron CR, Pondenis $H$, Stewart MC: Effect of fibroblast growth factor-2 on equine mesenchymal stem cell monolayer expansion and chondrogenesis. Am J Vet Res 2007, 68:941-945.

40. Buckley CT, Kelly DJ: Expansion in the presence of FGF-2 enhances the functional development of cartilaginous tissues engineered using infrapatellar fat pad derived MSCs. J Mech Behav Biomed Mater 2012 11:102-111.

41. Weiss S, Hennig T, Bock R, Steck E, Richter W: Impact of growth factors and PTHrP on early and late chondrogenic differentiation of human mesenchymal stem cells. J Cell Physiol 2010, 223:84-93.

42. Dickhut A, Dexheimer V, Martin K, Lauinger R, Heisel C, Richter W: Chondrogenesis of human mesenchymal stem cells by local transforming growth factor-beta delivery in a biphasic resorbable carrier. Tissue Eng Part A 2010, 16:453-464.

43. Park H, Temenoff JS, Tabata Y, Caplan Al, Raphael RM, Jansen JA, Mikos AG: Effect of dual growth factor delivery on chondrogenic differentiation of rabbit marrow mesenchymal stem cells encapsulated in injectable hydrogel composites. J Biomed Mater Res A 2009, 88:889-897.

44. Zhao L, Hantash BM: TGF-beta1 regulates differentiation of bone marrow mesenchymal stem cells. Vitam Horm 2011, 87:127-141.

45. Chase LG, Yang S, Zachar V, Yang Z, Lakshmipathy U, Bradford J, Boucher SE, Vemuri MC: Development and characterization of a clinically compliant xenofree culture medium in good manufacturing practice for human multipotent mesenchymal stem cells. Stem Cells Trans/ Med 2012, 1:750-758.

46. Lindroos B, Boucher S, Chase L, Kuokkanen H, Huhtala H, Haataja R, Vemuri $M$, Suuronen R, Miettinen S: Serum-free, xeno-free culture media maintain the proliferation rate and multipotentiality of adipose stem cells in vitro. Cytotherapy 2009, 11:958-972.

47. Wagey $R$, Short $B$ : Isolation, enumeration, and expansion of human mesenchymal stem cells in culture. Methods Mol Biol 2013, 946:315-334.

48. Dexheimer $V$, Mueller S, Braatz F, Richter W: Reduced reactivation from dormancy but maintained lineage choice of human mesenchymal stem cells with donor age. PLoS One 2011, 6:e22980.

49. Hagmann S, Moradi B, Frank S, Bäsig A-M, Dreher T, Richter W, Gotterbarm T: Chondrogenic and immunophenotypic properties of mesenchymal stem cells from osteoarthritis patients (abstract). Osteoarthritis Cartilage 2011, 20(Suppl. 1):S277.

50. Phinney DG, Kopen G, Righter W, Webster S, Tremain N, Prockop DJ: Donor variation in the growth properties and osteogenic potential of human marrow stromal cells. J Cell Biochem 1999, 75:424-436.

doi:10.1186/1471-2474-14-223

Cite this article as: Hagmann et al:: Different culture media affect growth characteristics, surface marker distribution and chondrogenic differentiation of human bone marrow-derived mesenchymal stromal cells. BMC Musculoskeletal Disorders 2013 14:223.

\section{Submit your next manuscript to BioMed Central and take full advantage of:}

- Convenient online submission

- Thorough peer review

- No space constraints or color figure charges

- Immediate publication on acceptance

- Inclusion in PubMed, CAS, Scopus and Google Scholar

- Research which is freely available for redistribution 